\title{
Color constancy of color reproductions in art paintings
}

\author{
Kinjiro Amano, ${ }^{1, *}$ (1) JoÃo M. M. Linhares, ${ }^{2}$ and Sérgio M. C. Nascimento ${ }^{2}$ \\ ${ }^{1}$ School of Electrical and Electronic Engineering, University of Manchester, Manchester M13 9PL, UK \\ ${ }^{2}$ Centre of Physics, University of Minho, Gualtar Campus, 4710-057 Braga, Portugal \\ ${ }^{*}$ Corresponding author: k.amano@manchester.ac.uk
}

Received 30 November 2017; revised 24 February 2018; accepted 27 February 2018; posted 28 February 2018 (Doc. ID 314643 ); published 29 March 2018

\begin{abstract}
Popular color reproductions of art paintings such as postcards are intended to remind viewers of the original works. It is, however, unclear how well the quality of the reproductions is preserved under various illuminations. Color constancy of the reproductions in relation to colors in the original paintings was estimated computationally with hyperspectral images of 15 th-century Flemish paintings, 20th-century modern abstract paintings, and their corresponding postcards with a series of illuminants: the CIE daylight D65 with correlated color temperature (CCT) $6500 \mathrm{~K}$, daylight D40, fluorescent lamps F2 and F11, and a LED lamp designed for museums with CCT approximately 3500-4000 K. Despite large colorimetric differences between the types of art paintings and between the illuminants simulated, local areas showed good color constancy: skin areas in the Flemish paintings ranged from 0.76 to 0.81 , whereas nonskin areas ranged from 0.19 to 0.68 . This result suggests that viewers may be able to achieve color constancy with the reproduction postcards by disregarding inconsistent colors representations from the original paintings caused by changes in illumination conditions. (02018 Optical Society of America
\end{abstract}

OCIS codes: (330.1690) Color; (330.1710) Color, measurement; (330.1715) Color, rendering and metamerism; (330.1720) Color vision.

https://doi.org/10.1364/JOSAA.35.00B324

\section{INTRODUCTION}

It is common that visitors at museums or art galleries purchase the reproduction of art paintings in the form of postcards. Color reproductions on the postcards are intended to remind viewers of the original works and viewers' appreciation of their colors. It is, however, unclear how good the quality of the reproduction is.

Since colors usually provide a signature of the painting and express emotions of the subject in the paintings, the quality of the color reproduction should be carefully managed. For example, skin coloration in the reproductions is crucial in capturing the color features of the original painting.

The postcards can be observed under any viewing conditions, whereas the original art painting is observed under a controlled environment, in particular, the illumination. Even with such differences in the viewing environment, can viewers obtain similar appreciation of colors in the original art painting with the postcard?

This question can be rephrased by the notion of color constancy. Color constancy refers to the effect where the perceived or apparent color of a surface remains constant despite changes in the illumination, which may have different spectral composition [1]. Thus, the question to address in this study is how well color appearance is preserved on the reproduction postcard under different illuminations when compared with the original paintings.

Two types of art paintings and corresponding postcards were selected to evaluate the color constancy: two of 15 th-century Flemish paintings and three of 20th-century modern paintings, (Fig. 1). The corresponding postcards were obtained at the museums where the original paintings belonged. Each of the two Flemish paintings included skin areas of human faces and hands. One of the paintings represents joy and happiness, with a lady holding an infant, and another represents sadness, with a lady holding a deceased male. These emotions might have been expressed by elaborate use of color tone and shade. In contrast, the three modern paintings are almost abstract and consist of color patches with various shapes and colors. Thus, there is no explicit representation of emotion in the modern paintings.

The hyperspectral images of the original paintings and the corresponding postcards were used to obtain precise color rendering of the surfaces under spectrally different illuminations with which colorimetric analysis were undertaken. The use of the hyperspectral imaging for the color vision sciences has been reported elsewhere [2-4]. 

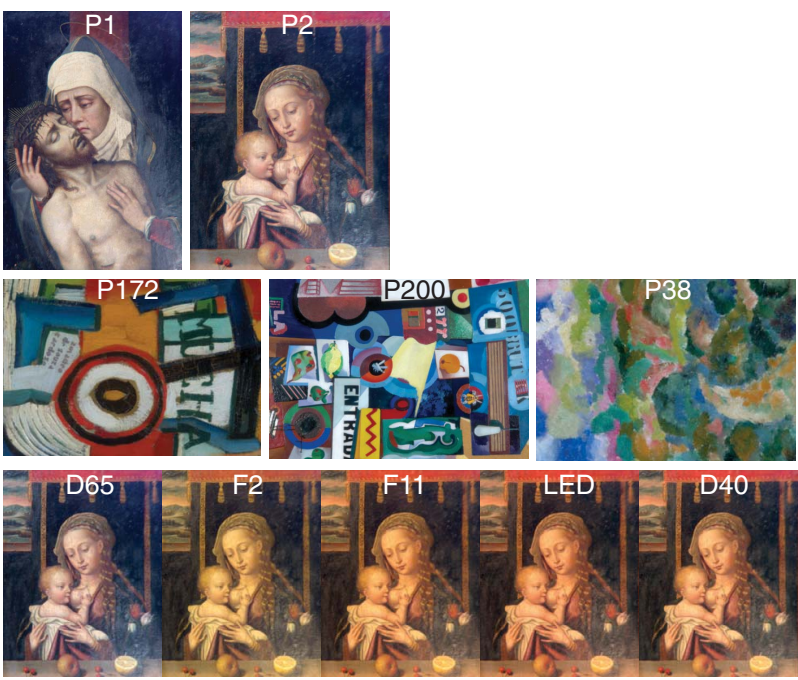

Fig. 1. Sample images of the art paintings. The first and second rows show two Flemish paintings (P1 and P2) and three modern paintings by Amadeo de Souza-Cardoso (P172, P200, and P38), respectively. The color images were generated from the hyperspectral data with illuminant D65. The images in the third row show the color renderings with different illuminants (D65, F2, F11, LED, and D40) on the Flemish painting P2.

Since there is a large interval between the creation dates of the Flemish and modern paintings, it is very likely that color pigments used in these paintings could be physically different, whereas color pigments in the modern paintings may be closer to those used in the color printing of the postcards. It is the spectral properties by the hyperspectral imaging that enables us to estimate the differences and to lead to colorimetric analysis. The pigments in the modern paintings have been analyzed for the purpose of the conservation process elsewhere [5].

The designs of lightings for art paintings at museums and galleries vary among the institutions, although they follow the standards [6-8]. The correlated color temperature (CCT) of the lightings is usually approximately $4000 \mathrm{~K}$ or lower ([7], cf. [9]). The use of LED lamps at the museums has increased rapidly, along with the technological advancement, because of their advantages in the stability of light emission, performance of color rendering, and energy consumption [10]. In contrast, the postcards could be observed under any type of illumination, even under direct sunlight or bright daylight.

Considering these facts, the illuminants simulated in this study were the Commission Internationale de l'Eclairage (CIE) standard illuminant D65 with CCT $6500 \mathrm{~K}$, fluorescent lamps F2 and F11 with CCT approximately $4000 \mathrm{~K}$, and an LED lamp designed for the art materials with CCT approximately $3500 \mathrm{~K}$ and a daylight D40 with CCT $4000 \mathrm{~K}$.

Color constancy of human vision has been studied mainly in psychophysical experiments, for example, with color matching tasks or operational judgments $[1,11,12]$. Observers' performance in color constancy tasks has been usually estimated by a standard color constancy index [11]. The same principle is applicable to estimate color constancy computationally. Here, by introducing the reproduction color constancy index (RCCI) $[13,14]$, color constancy between the original paintings and the reproduction postcards under varied illuminants was estimated. The RCCI is defined by regarding the colors on the postcards as an estimate of observer's matched colors, then applying the calculation of the standard color-constancy index. The index unity represents perfect constancy and the lower the index, the greater the error.

Mean RCCI over the local areas selected was as high as 0.81 under illuminants with lower CCT. The result suggests that viewers can achieve fairly good color constancy, although there are variations between the local areas and the type of paintings. The levels are close to the color constancy indices achieved by human psychophysics [1].

Spectral and colorimetric properties such as chroma and lightness between the original paintings and the postcards and between the illuminants were analyzed as the course of the variations in RCCI. Statistics of color properties have been quantified by estimating chromatic contrast and color difference (e.g., [15]). Depending on the type of paintings, the characteristics in the transformation of chromaticity distributions (color gamut) were different (e.g., $[16,17])$. The Flemish paintings had an expansion of color gamut between the media, as if the effects of memory color and color preference (e.g., $[18,19])$ were taken into account. But the modern paintings had an opposite effect. These results suggest that, despite the colorimetric differences, viewers may be able to achieve good color appreciation by the reproductions of the original art paintings.

\section{METHODS}

\section{A. Materials}

Two of the 15th-century Flemish paintings belonged to the Edição do Museu Nogueira Da Silva, Braga, Portugal, and three of the modern paintings by Amadeo de Souza-Cardoso (18871918) belonged to the Museu Calouste Gulbenkian, Lisbon, Portugal. The Flemish paintings, Senhora com Cristo Morto (a close English translation may be Lamentation over the Body of Christ) and Senhora do Leite da Meia Laranja (a close English translation is Madonna with Child), were dated as 15th century and were made by unknown painter(s) ("desconhecido" in Portuguese). For the modern paintings by Amadeo de SouzaCardoso, since the titles of the paintings were not given formally, we use the labels P172, P200, and P38. The labels were based on the catalog [20] (it should be noted that the museum has another set of coding for the three paintings, 86P21, 77P9, and $77 \mathrm{P} 3$, respectively). Each of the paintings was dated approximately 1915, 1917, and 1912, respectively. Detailed information on the paintings of Amadeo de Souza-Cardoso and the procedure of hyperspectral imaging has been given elsewhere [20,21]. The sample images rendered under D65 are shown in Fig. 1 (the first and second rows).

In the manufacturing process of the postcards, it is common that the images of the original painting can be adjusted or the edges of the image can be removed so that the image can fit to the postcard format. In this study, the images of the original modern paintings were cropped so that the image size can be almost the same as the image content of the corresponding postcards (the original sizes were P172, $21.4 \mathrm{~cm} \times 27.3 \mathrm{~cm}$; P200, $93.5 \mathrm{~cm} \times 75.5 \mathrm{~cm} ; \mathrm{P} 38,33.9 \mathrm{~cm} \times 27.4 \mathrm{~cm}$ ). Since the details of the adjustments made in the manufacturing 
process are unknown, the areas of the removals were made by authors' judgments.

The final images of the original paintings and the postcards had different pixel resolution. Therefore, instead of pixel-bypixel comparison, analyses in this study were mainly made on local areas chosen to cover almost the same image contents between the original painting and the postcard.

This study considered only surface colors on the twodimensional digitized image of the paintings and postcards. There may be often three-dimensional geometrical structures, such as a bump of paint or brush strokes, on the real paintings. Those may well create an effect of immersiveness in the real paintings but were not of concern in this study, as they were not represented in the postcards.

\section{B. Hyperspectral Imaging and Reflectance Estimation}

The hyperspectral image of the paintings and the postcards was acquired by a custom-built hyperspectral imaging system. The technical details of the system and imaging procedure have been reported elsewhere $[21,22]$. Some of the essential details are given here.

The system consisted of a low-noise Peltier-cooled monochromatic digital camera (Hamamatsu, model C4742-9512ER, Hamamatsu Photonics K. K., Japan) and a tunable liquid crystal filter (Varispec, model VS-VIS2-10-HC-35SQ, Cambridge Research \& Instruments, Inc., MA). The wavelengths of the hyperspectral imaging ranged over visible range from 400 to $720 \mathrm{~nm}$ in $10 \mathrm{~nm}$ intervals. FWHM of the tunable filter is $10 \mathrm{~nm}$ at $550 \mathrm{~nm}$. The spatial resolution of the camera was 1344 pixels $\times 1024$ pixels. A small neutral surface (Munsell N7) was placed in the scene of the artifacts at the image acquisition to serve as a spectral reference to process the data to estimate the spectral surface reflectances.

Each of the paintings and postcards was imaged separately. Thus, the hyperspectral image acquisitions of the original paintings were undertaken at each of the museums, and those of the postcards were undertaken at a specially designed laboratory at the University of Manchester. The image size of the real Flemish paintings subtended approximately 960 pixels $\times$ 690 pixels for the original paintings and 785 pixels $\times$ 570 pixels for the postcards; the modern abstract paintings subtended approximately 1120 pixels $\times 770$ pixels for the original and 820 pixels $\times 570$ pixels for the postcard.

\section{Illuminant Simulation}

Colors under the four illuminations as well as the CIE standard daylight D65 were calculated. The simulated illuminants were two types of the CIE fluorescent illuminants F2 and F11, an LED lamp designed for art materials, and the daylight D40.

Illuminant D65 corresponds to average noon daylight with a CCT of approximately $6500 \mathrm{~K}$ and is defined as the standard illuminant for colorimetric calculations [23]. The fluorescent illuminant F2 is a common fluorescent lamp used for typical office illumination and is "cool white fluorescent," with a CCT of approximately $4200 \mathrm{~K}$. Another fluorescent illuminant, F11, has three narrow peaks over the visible spectrum [24] with a CCT of approximately $4000 \mathrm{~K}$ and a color rendering index (CRI) of approximately 83 , which is mainly used as warehouse lighting. The LED lamp was specially designed for art materials (model XSM9535-1000-C, Xicato Inc., San Jose, CA; spectral data: http://www.xicato.com), whose CCT is approximately $3500 \mathrm{~K}$. The difference from the conventional LED lamp is the fact that it does not have any strong power peak on the shorter wavelength, which is considered to harm color pigments. Illuminant D40 is close to a domestic incandescent or tungsten lamp. The spectral profile of the daylight D40 was obtained from three daylight spectral basis functions [23]. The spectral profiles of the fluorescent lamps were obtained from the Rochester Institute of Technology data set [25] in a range from 400 to $720 \mathrm{~nm}$ in $10 \mathrm{~nm}$ intervals. Luminance of each illuminant was simulated to be constant, in this study, set to $100 \mathrm{~cd} / \mathrm{m}^{2}$. The sample images of the color appearance under the different illuminants on the Flemish painting P2 are shown in the third row in Fig. 1.

\section{Local Areas}

Each of the two Flemish paintings includes two human figures. Coloration of human skin is of interest because it often represents an individual's emotion, which might correspond to the main theme of the painting. To compare the color appearance of skin areas between the original paintings and the postcards, local skin areas of forehead, cheek, and hand (or shoulder if any hand was not available) were selected from each figure (Fig. 2). These selections of the local areas were similar to those used in a data set of human skin color [26]. The other nonskin areas were also selected (Fig. 2). The local areas selected in the modern paintings are shown in Fig. 3. The local areas were selected pseudo-randomly but restricted so as not to have any nonuniformity of colors within the area.
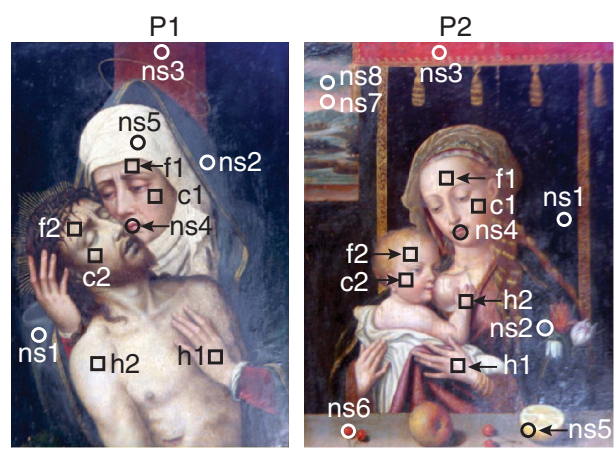

Fig. 2. Local areas on the two Flemish paintings $P 1$ and P2. Local skin areas, forehead, cheek, and hand/shoulder are indicated by $f, c$, and $h$ with squares, respectively; nonskin local areas are indicated by ns with circles. The sizes of the squares and circles were adjusted for visibility.
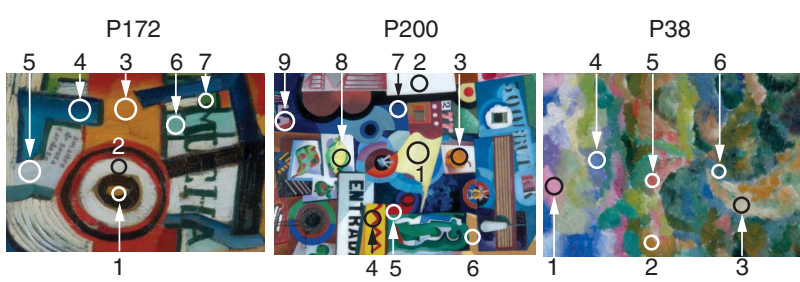

Fig. 3. Local areas selected on the modern paintings. Areas selected are indicated by circles. The sizes of circles were adjusted for visibility. 
The size of each local area varied across the areas, ranging from 12 pixels on the lips area (area ns4 in Fig. 2) to over 11,600 pixels (area ns3 in Fig. 2) in the Flemish paintings; from 160 pixels (area 5 in P38 in Fig. 3) to over 11,400 pixels (area 2 in P200 in Fig. 3).

\section{E. Colorimetric Analysis}

Colorimetric analysis was undertaken on the colors of the local areas in the original paintings and corresponding postcards under the simulation of different illuminations (Subsection 2.C). Chromaticity coordinates were calculated in the CIELAB space. To estimate saturation of colors, chroma was defined with $a^{*}$ and $b^{*}$ coordinates:

$$
\text { Chroma }=\sqrt{a^{* 2}+b^{* 2}} \text {. }
$$

\section{F. Reproduction Color Constancy Index}

Color-constancy performance has been assessed by the RCCI $[13,14]$, which is defined analogously to the standard colorconstancy index established in the vision sciences $[1,11]$. Thus, in a uniform color space CIELAB, where the chromaticity coordinates of the local areas selected in an original painting and the corresponding areas in the postcard were located, let $s$ be the Euclidian distance between the color of the original painting and color of the postcard under a test illuminant and $t$ be the Euclidian distance between the original painting under the reference and test illuminations. Then, the RCCI is $1-s / t$. Perfect constancy corresponds to unity, and the index is lower for a higher error level. Illuminant D65 was either the reference or test illuminant. That is, the directions of the illumination changes between the reference and test were considered, one of which is always paired with D65.

\section{RESULTS}

\section{A. Spectral Analysis}

Spectral reflectances on the selected local skin areas in the Flemish painting P2 are shown in Fig. 4.

Skin areas in the original painting and the postcard are shown in Figs. 4(a) and 4(b), respectively. There are clear differences in the spectral profiles between the original painting and the postcard. Spectra of the original painting are smoother than those of the postcards. The surface reflectances of the original paintings were almost $50 \%$ lower than the postcard,

(a)

(b)
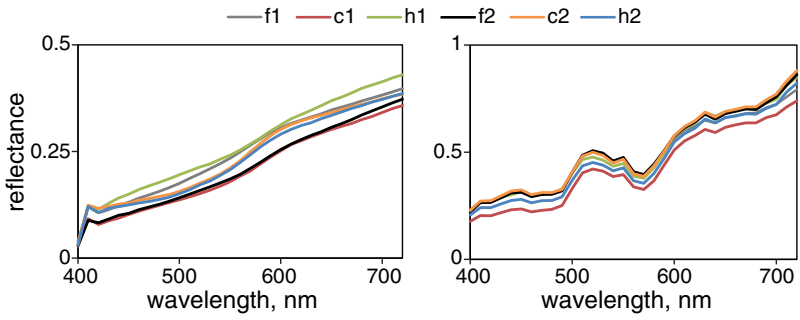

Fig. 4. Spectral reflectances of the Flemish painting P2. (a) Original painting and (b) postcard. The local skin areas, forehead, cheek, and hand/shoulder $(f, c$, and $h$, respectively) correspond to those shown in Fig. 2. indicating the original has darker color than the postcard when illuminated by the same light source.

The evaluation of the spectral differences between two spectra, for example, one from the original paintings and another from the postcard, can be performed with root mean square error (RMSE) and spectral similarity value (SSV) [Eqs. (7)-(9) in Ref. [24]]. Thus, let the spectrum of the original painting denote $r_{r}$ and the spectrum from the postcard $r_{t}$ :

$$
\text { RMSE }=\sqrt{\frac{1}{n} \sum_{i=1}^{n}\left(r_{r, i}-r_{t, i}\right)^{2}},
$$

$$
\mathrm{SSV}=\sqrt{\mathrm{RMSE}^{2}+S^{2}}
$$

where $i$ is the $i$ th spectral component of reflectances, and $n$ is the total number of components. $S^{2}$ is defined by

$$
S^{2}=1-\left[\frac{\frac{1}{n} \sum_{i=1}^{n}\left(r_{r, i}-\mu_{r}\right)\left(r_{t, i}-\mu_{t}\right)}{\sigma_{r} \sigma_{t}}\right]^{2},
$$

where $\mu_{r}$ and $\mu_{t}$ are the means of the reflectances of the original painting and the postcard, respectively, computed over wavelength, and $\sigma_{r}$ and $\sigma_{t}$ are the standard deviations of the reflectances of the original painting and the postcard, respectively.

The values of RMSE and SSV between the original painting and the postcards were similar to those reported in the similar comparisons [13], where the original human skin spectra were compared with artificial facial skin with a calibrated additive manufacturing process. Overall, of skin areas and nonskin areas in both paintings, RMSE and SSV were not correlated $\left(R^{2}=0.54\right)$. Since SSV includes RMSE in its definition, the difference was caused by the covariance term between the original painting and the postcard [Eq. (4)].

Spectral reflectances on the local areas in the modern painting, P200, are shown in Figs. 5(a) and 5(b). Similar to the Flemish paintings, the reflectance spectra of the original painting was smoother than the postcard. However, the level of reflectances was close to each other. The mean values of RMSE and SSV were similar to those for the Flemish paintings. There are bumps on the spectra of postcards in both paintings, approximately 480 and $580 \mathrm{~nm}$. These may be originated by the color inks in the manufacturing process. Similar characteristics have been reported in the additive manufacturing of colored skin $[13,14]$.

The correlation of the RMSE and SSV to reproduction color constancy RCCI is considered in Section 4. (a)

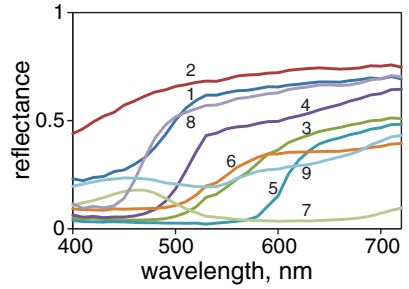

(b)

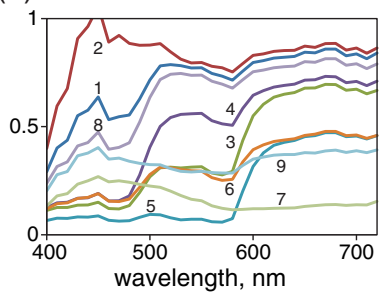

Fig. 5. Spectral reflectances of the selected local areas in the modern painting P200. (a) Original painting and (b) postcard. The numbers correspond to the areas selected, as shown in Fig. 3. 


\section{B. Colorimetric Analysis}

Chromaticity coordinates $L^{*}, a^{*}$, and $b^{*}$ in the CIELAB space at each local area were calculated by applying the illuminants of the daylights D65 and D40, fluorescent lamps F2 and F11, and an LED lamp (Subsection 2.C). The chromaticity of each local area was defined by the spatial average within each local area.

The chromaticities of the skin areas in the two original Flemish paintings under daylight (D65) are shown in the CIELAB $\left(a^{*}, b^{*}\right)$ coordinates in Fig. 6(a) and (chroma, $\left.L^{*}\right)$ in Fig. 6(b), respectively.

Skin colors in the Flemish painting P1 may have represented the emotion of sadness, while the painting P2 may have represented joy and happiness (Subsection 2.A). Those emotions seemed to be reflected in the chromaticities; that is, the chromaticities of the skin areas in the painting P1 located toward greenish for sadness (lower $a^{*}$ ), and those in the P2 toward more reddish (higher $a^{*}$ ) for joy [Fig. 6(a)], whereas the lightness of these areas is almost constant [Fig. 6(b)].

Figures 6(c) and 6(d) show the shift of chromaticities under different illuminants, in the CIELAB $\left(a^{*}, b^{*}\right)$ coordinates in Fig. 6(c) and (chroma, $L^{*}$ ) in Fig. 6(d), respectively. Differences in the influence of the illuminants between the original and the postcard can be observed by comparing filled and open symbols. Arrows indicates the shifts of chromaticities due to the change of illuminants (from D65 to D40 or lower CCT).

(a)

(b)

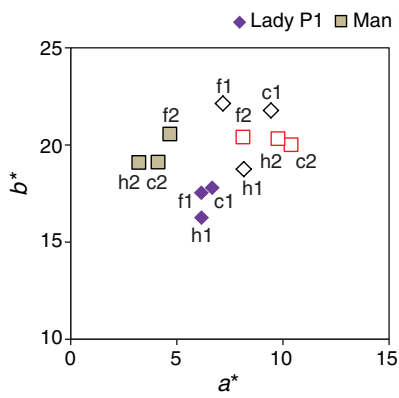

(c)

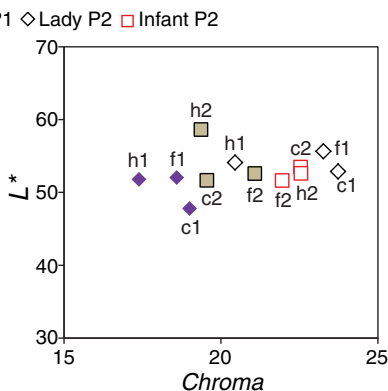

(d)
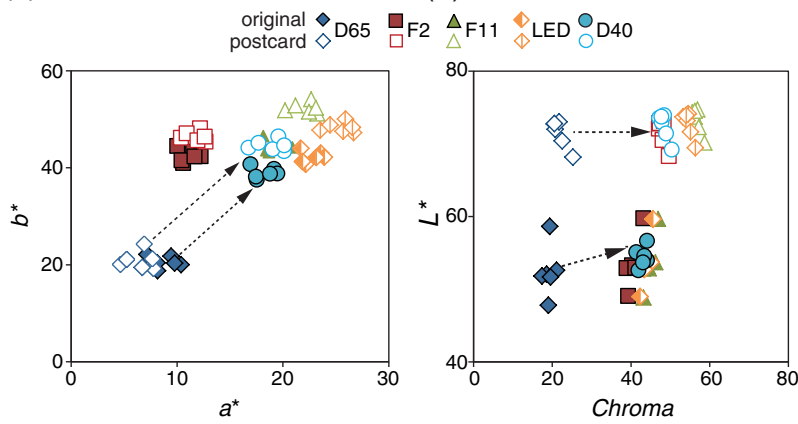

Fig. 6. Chromaticities of the local skin areas in the Flemish paintings P1 and P2. (a) Comparison of chromaticities of the skin areas in the Flemish paintings P1 and P2 under D65 on $\left(a^{*}, b^{*}\right)$ and $(\mathrm{b})$ on (chroma, $L^{*}$ ) space in the CIELAB. Labels $f, c$, and $h$ correspond to those in Fig. 2; (c) comparisons of chromaticities of the skin areas under the different illuminants on $\left(a^{*}, b^{*}\right)$ and (d) (chroma, $\left.L^{*}\right)$ in the CIELAB. Filled symbols show the chromaticities in the original paintings and open symbols show those in the postcards. Arrows indicate the shifts of chromaticities due to the change of illuminants (from D65 to D40 or lower CCT).
The chromaticities of the postcards [open symbols in Figs. 6(c) and 6(d)] were similar to those in the original painting under the illuminant D65. However, the lightness levels are much higher, as expected from the reflectance profile in Fig. 4 (Subsection 3.A). These characteristics were altered under different illuminations with the lower CCT, i.e., fluorescent lamps F2 and F11, an LED lamp and daylight D40, (Subsection 2.C). The chroma of the postcards was higher than that of the original paintings, while the lightness of the postcards was constantly higher than the original across the illuminants. On the nonskin areas, as with the skin areas (figure not shown), the chromaticities in the postcards have higher saturation and lightness than the original.

The shifts of chroma of the skin colors between the original painting and the postcards of the Flemish paintings under the illuminants with the lower CCT (F2, F11, LED, or D40) are markedly larger. With the Flemish paintings P1 and P2, for example, the mean differences in chroma over skin areas between the original and postcard were 6.4 and 8.8 units under illuminant F11, respectively. The increment of the chroma was also observed on the nonskin local areas in the Flemish paintings. Under illuminant F11, the mean differences over the areas were 6.6 and 10.8 units in each of the paintings, respectively. The mean difference in chroma between the original and postcard over the illuminants with lower CCT and local areas are 4.3 and 6.1 units for $\mathrm{P} 1$ and $\mathrm{P} 2$, respectively.

Two of the modern paintings (P172 and P200) had different characteristics from the Flemish paintings. The chromaticities on the local areas in the postcards were desaturated, and chroma was smaller compared with those on the original paintings, while lightness was almost constant under the illuminants with lower CCT. Figure 7 shows the chromaticities $\left(a^{*}, b^{*}\right)$ and (chroma, $L^{*}$ ) of the painting P200. Filled symbols in Figs. 7(a) and 7(b) represent the chromaticities of the local areas in the original painting, and open symbols in Figs. 7(c) and 7 (d) represent the corresponding areas in the postcard; different symbols indicate different illuminants. The numbers inserted into diamond symbols D65 correspond to the areas shown in Fig. 2. Differences in the influence of the illuminants between the original and the postcard can be observed by comparing filled and open symbols in Figs. 7(a), 7(c), and 7(b), 7(d), respectively. Arrows indicates the shifts of chromaticities caused by illuminants (from D65 to D40 and lower CCT). The influence of the illuminants on the magnitudes and direction of the shifts varied across the local areas.

The direction of the chroma shift was different between the Flemish and modern paintings. For the three modern paintings, the decrements in chroma from the original paintings averaged over the illuminants with the lower CCT; F2, F11, LED, and D40 were $-6.3,-9.5$, and -5.2 units, respectively. However, a reverse trend appeared for D65. The chroma of the original painting is higher than that of the postcard. These variations of characteristics may originate in complex interactions of the surface reflectances and the spectra of the illuminants.

These shifts are in fact not limited in the local areas selected, but also the whole of colors in an image. This issue is to be discussed in Subsection 3.D. 
(a)

(b)

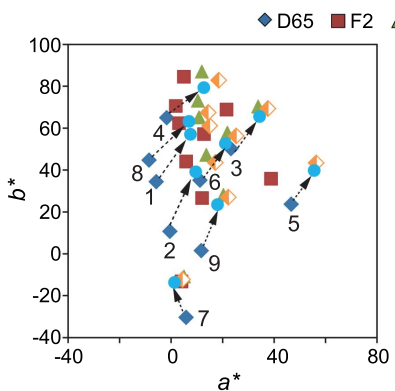

(c)

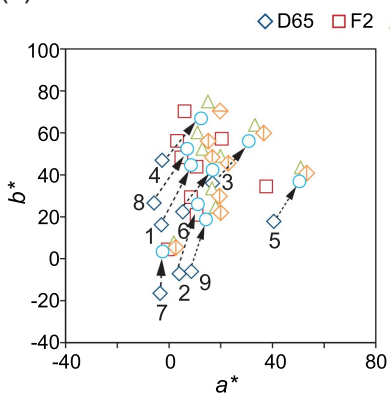

(d)
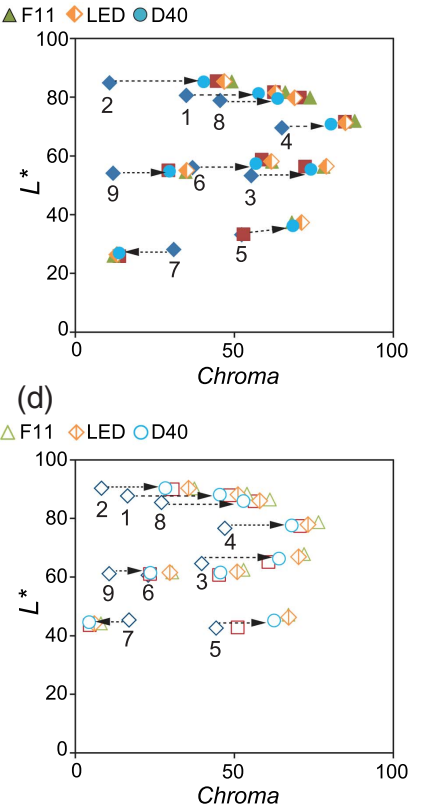

Fig. 7. Chromaticities of the local areas in the modern painting P200. The colors of the symbols indicate the difference of the illuminants. (a) Comparison of chromaticites of the local areas in the original painting under the different illuminants (D65, F2, F11, LED, and D40) on $\left(a^{*}, b^{*}\right)$ and (b) on (chroma, $\left.L^{*}\right)$ in the CIELAB; (c) comparisons of chromaticities on the local areas in the postcard under the different illuminants on $\left(a^{*}, b^{*}\right)$ and $(\mathrm{d})$ on (chroma, $\left.L^{*}\right)$ in the CIELAB. The numbers inserted into diamond symbols D65 correspond to the areas shown in Fig. 2. Arrows indicate the shifts of chromaticities caused by illuminants (from D65 to D40 and lower CCT).

\section{Reproduction Color Constancy}

The performance of color constancy was evaluated by the RCCI (Subsection 2.F). Here, two-dimensional $\left(a^{*}, b^{*}\right)$ color space was considered because of the large difference in the lightness $\left(L^{*}\right)$ between the original painting and the postcard, in particular, the Flemish paintings (Fig. 4).

The RCCI on the skin areas in the Flemish paintings was relatively higher than the nonskin areas, except for the daylight D40. The average value over the skin areas (forehead, cheek, and hand in the two individuals in a painting) on the Flemish painting P2 ranged from 0.76 to 0.84 , with a rank of the illuminants with the RCCI low to high, F11, LED, and F2. RCCI with D40 was significantly low, with 0.22 (Fig. 8). Similar levels appeared with the Flemish painting P1, ranging from 0.78 to 0.84 with the same rank of the illuminants, whereas the RCCI was approximately 0.18 with D40. The RCCIs on the nonskin areas were slightly lower than on the skin areas. Among the selected nonskin local areas, the RCCI ranged from 0.53 to 0.76 over the illuminants F2, F11, and LED. The RCCI with $\mathrm{D} 40$ was down to a negative value, possibly because of the irregularity of color transformation.

The RCCIs on the local areas in the modern paintings were lower than in the Flemish paintings. Mean RCCI over the local areas under different illuminants (excluding D40) ranged from 0.19 to 0.38 in P172, from 0.40 to 0.59 in P200, and from

(a)

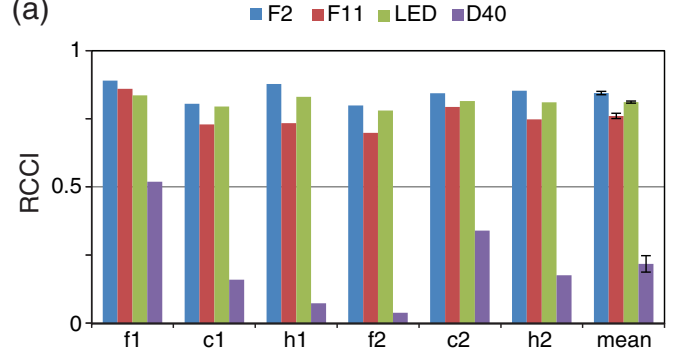

(b)

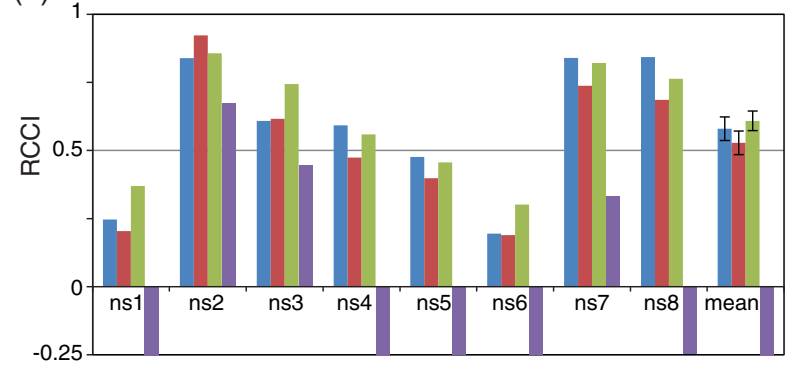

Fig. 8. RCCI of (a) local skin areas and (b) nonskin areas in the Flemish painting P2. To compute RCCI, the illuminant D65 was paired with one of the illuminants F2, F11, LED, or D40. The RCCIs with negative values were cut off at -0.25 . Error bars represent standard error of mean. The labels correspond to the areas indicated in Fig. 2.

0.59 to 0.68 in P38, respectively. RCCI varied largely across the local areas. Figure 9 shows the RCCI for the painting P200.

Nevertheless, the reproduction color constancy obtained here is not so different from those achieved in color-constancy experiments by human observers [1]. In particular, the RCCIs of the skin areas were relatively higher. But the RCCI on the nonskin areas had a large variation, depending on the chromaticity of the local areas. Thus, RCCI seemed to be influenced by the irregularity in the transformation of chromaticities across the illuminants.

\section{Color Gamut}

Distributions of chromaticities represented with $\left(a^{*}, b^{*}\right)$ coordinates in the CIELAB space, color gamut, and with (chroma, $\left.L^{*}\right)$ coordinates under the illuminants D65 and LED are compared in Fig. 10 for one of the Flemish paintings P2 and one of the modern paintings P200, respectively. Figures 10 (a) and 10(c) indicate the plots of $\left(a^{*}, b^{*}\right)$ and Figs. $10(\mathrm{~b})$ and $10(\mathrm{~d})$ indicate (Chroma, $L^{*}$ ) in the CIELAB.

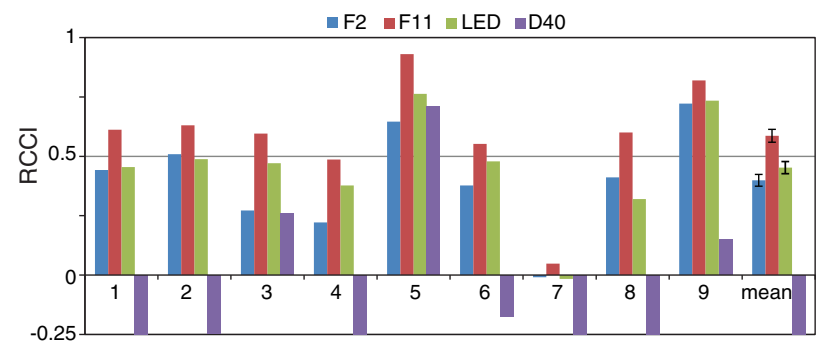

Fig. 9. RCCI on the local areas in the modern painting P200. The RCCIs with negative values were cut off at -0.25 . Error bars represent standard error of mean. The labels correspond to the areas indicated in Fig. 3. 

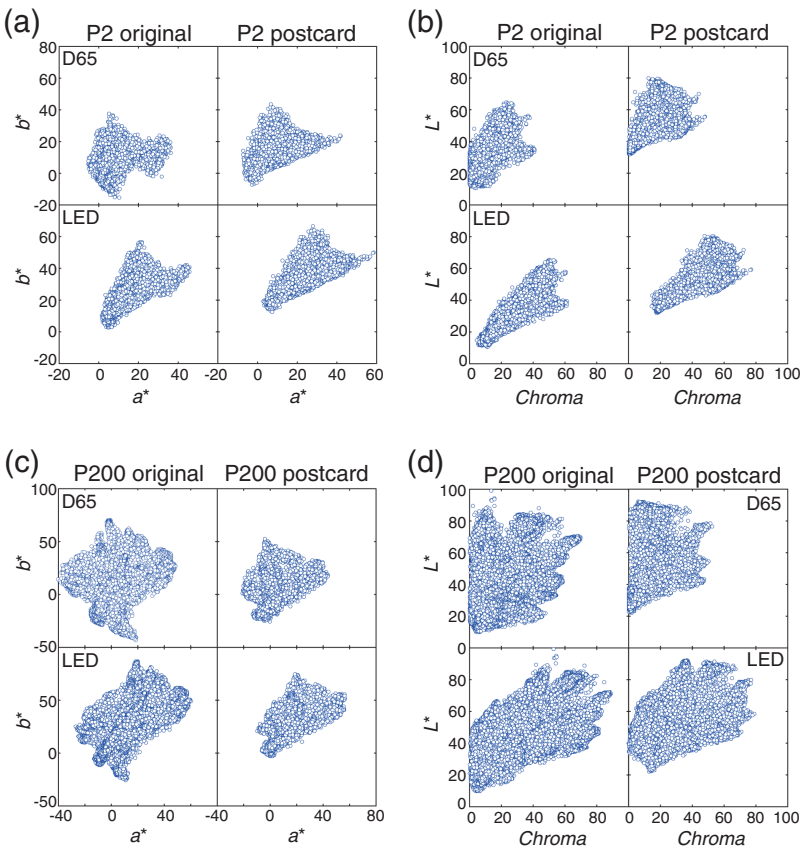

Fig. 10. Transformation of color gamut on (a) and (b) the Flemish P2 and (c) and (d) modern painting P200 under the illuminants D65 and LED, respectively. Color gamuts of the original painting and the postcard are shown in (a) and (c) on $\left(a^{*}, b^{*}\right)$ and (b) and (d) on (chroma, $L^{*}$ ) in the CIELAB, respectively.

The color gamut of the original paintings is markedly different from those of the postcards in both types of the paintings, as seen in Fig. 11. Under the illuminant D65, for the Flemish painting P2, the color gamut of the postcard appeared to be elongated toward higher $a^{*}$ (reddish) than the original painting. For the modern painting P200, the gamut of the postcard is much smaller than that of the original. Along with the different illuminants with lower CCT, the gamut for P2 tended to extend toward higher $a^{*}$, as expected, and the gamut of the original paintings became rotated and skewed.

(a)

(b)
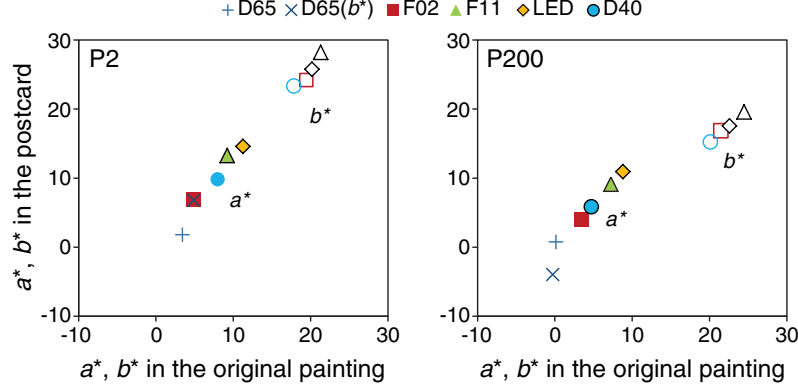

Fig. 11. Skewness caused by the transformation of color gamut in (a) the Flemish painting P2 and (b) the modern painting P200. Median chromaticities of $a^{*}$ and $b^{*}$ distributions of the original painting are shown as a function of those in the postcard along with the different illuminants, D65, F2, F11, LED, and D40. The ordinate and abscissa are the chromaticities of the original paintings and those of the postcard, respectively. The rank of the illuminants is different between $a^{*}$ and $b^{*}$.
From (chroma, $L^{*}$ ) coordinates, the difference in lightness distribution is clear. The distribution in the postcards has higher lightness and chroma compared with the original paintings.

The difference in the size of the color gamut can be quantified by the chromatic contrast, i.e., color difference from the median chromaticity of $\left(a^{*}, b^{*}\right)$ in the CIELAB space. Let $C$ indicate chromatic contrast; $C$ is defined as

$$
C=\sqrt{\left(L^{*}-L_{\text {med }}^{*}\right)^{2}+\left(a^{*}-a_{\text {med }}^{*}\right)^{2}+\left(b^{*}-b_{\text {med }}^{*}\right)^{2}},
$$

where $a_{\mathrm{med}}^{*}, b_{\mathrm{med}}^{*}$ and $L_{\mathrm{med}}^{*}$ represent the median chromaticity of an image.

Table 1 lists the ratio of mean $C$ over the local areas in the postcard under each illuminant to mean $C$ over the local areas in the original painting. If the ratio is larger than unity, it indicates the increment of the chromatic contrast from that of the original under D65. The ratios on the Flemish paintings are larger than unity regardless of the type of the local areas, whereas those in the modern paintings P172 and P200 are less than unity.

Averaged over skin and nonskin areas in each of the Flemish paintings, by analysis of variance without repetition for the five paintings and five illuminants, $C$ values over the five paintings and five illuminants, the difference between the paintings is significant $(F(4,16)>200, p=0.0)$; so is the difference between illuminants $(F(4,16)=6.2, p=0.0)$.

Instead of the local areas, $C$ can be computed for all points in a whole image. Table 2 lists the ratios of mean $C$ over all points on the postcard under each of the illuminants to mean $C$ over all points under D65 on the original paintings. If the ratio is larger than unity, it indicates that the color gamut is larger than the gamut of the original painting under D65. In Table 2, it is clear that the increment and decrement of the ratio varied with the type of paintings, i.e., the Flemish paintings and the modern paintings. The postcard of the Flemish paintings had almost 1.5 times larger gamut than the original under D65 illuminant, whereas those of the modern painting P200 had almost 0.6 times smaller. But the modern painting P38 seems to have larger gamut.

Analysis of variance without repetition for the five paintings and five illuminants showed that the effect of the

Table 1. Ratios of Chromatic Contrast on the Local Areas in the Postcard under Each of the Illuminants F2, F11, LED, and D40 to the Contrast in the Original Painting under Illuminant D65a

\begin{tabular}{|c|c|c|c|c|c|c|c|}
\hline \multirow{3}{*}{$\begin{array}{l}\text { Illuminant } \\
\text { on } \\
\text { Postcard } \\
\end{array}$} & \multicolumn{4}{|c|}{ Flemish Paintings } & \multicolumn{3}{|c|}{ Modern Paintings } \\
\hline & \multicolumn{2}{|r|}{ P1 } & \multicolumn{2}{|r|}{ P2 } & \multirow[b]{2}{*}{ P172 } & \multirow[b]{2}{*}{ P200 } & \multirow[b]{2}{*}{ P38 } \\
\hline & Skin & Nonskin & Skin & Nonskin & & & \\
\hline D65 & 1.104 & 1.108 & 1.081 & 1.095 & 0.859 & 0.848 & 1.029 \\
\hline $\mathrm{F} 2$ & 1.081 & 1.114 & 1.059 & 1.067 & 0.881 & 0.901 & 1.077 \\
\hline F11 & 1.131 & 1.128 & 1.089 & 1.131 & 0.880 & 0.950 & 1.106 \\
\hline LED & 1.135 & 1.124 & 1.077 & 1.115 & 0.876 & 0.930 & 1.089 \\
\hline D40 & 1.129 & 1.120 & 1.075 & 1.102 & 0.874 & 0.910 & 1.067 \\
\hline
\end{tabular}

${ }^{a}$ Chromatic contrast is defined by color difference from the median chromaticity $\left(a^{*}, b^{*}\right)$ in the CIELAB. The ratio greater than unity represents the expansion of chromatic contrast. 
Table 2. Ratios of Chromatic Contrast of the Postcard under Each of the Illuminants F2, F11, LED, and D40 to the Contrast of the Original Painting under Illuminant D65

\begin{tabular}{lccllll}
\multirow{2}{*}{$\begin{array}{l}\text { Illuminant on } \\
\text { Postcard }\end{array}$} & \multicolumn{2}{c}{ Flemish Paintings } & & \multicolumn{3}{c}{ Modern Paintings } \\
\cline { 2 - 3 } \cline { 5 - 6 }$n$ & P1 & P2 & & P172 & P200 & P38 \\
\hline D65 & 0.859 & 1.102 & & 0.802 & 0.595 & 1.101 \\
F2 & 1.189 & 1.248 & & 0.795 & 0.621 & 1.046 \\
F11 & 1.414 & 1.533 & & 0.950 & 0.729 & 1.154 \\
LED & 1.381 & 1.503 & & 0.935 & 0.716 & 1.139 \\
D40 & 1.286 & 1.434 & & 0.909 & 0.691 & 1.112 \\
\hline
\end{tabular}

${ }^{a}$ The ratio greater than unity represents the expansion of chromatic contrast.

illuminants was significant $(F(4,16)=7.73, p<0.05)$ and the difference between the paintings was significant $(F(4,16)=$ $47.8, p<0.05)$.

The shape and size of color gamut vary along with the illuminants F2, F11, and D40, despite their CCT being all approximately $4000 \mathrm{~K}$, and the LED with CCT being $3500 \mathrm{~K}$ (the results with D65, F2, and LED are shown in Fig. 11). The transformation of color gamut had not only the expansion but also skewed toward higher $a^{*}$ (reddish) and $b^{*}$ (yellowish), as expected $[15,23,27]$. The skewness of the color gamut is summarized by tracing the median chromaticity in each of $\left(a^{*}, b^{*}\right)$ coordinates under different illuminants.

The median chromaticity of $a^{*}$ and $b^{*}$ in the original painting under each of the illuminants against those in the postcard is shown in Figs. 11(a) and 11(b) for the Flemish painting P2 and the modern painting P200, respectively. The order of the illuminants in $a^{*}$ and $b^{*}$ coordinates is different, and their stepsize is uneven across the illuminants. The magnitude of the skewness depends on the paintings.

The color gamut of the common color inks for printing [cyan, magenta, yellow, and key (black), known as CMYK] is in general smaller than that of the colors available in standard digital devices with RGB (red, green, and blue) format, and the RGB gamut is smaller than for natural scenes and color pigments for the paintings (e.g., [21,28]). Such technical constrains may have provided a limit on color available between media, and, consequently, color constancy between the original paintings and the reproduction postcards. Transformation of color gamut across media, known as color gamut mapping, has been studied extensively $[16,17,29]$. However, as observed, the difference in colorimetric characteristics between the types of the paintings (e.g., Fig. 10), any of objective and scientific procedures, e.g., the gamut mapping, did not seem to be undertaken in the reproductions of the individual paintings used in this study. The colorimetric differences between the two types of paintings may indicate that the reproduction processes might have been different between the institutions. In general practice, the reproduction process consists of digital imaging, color adjustments, and printing. The color adjustments are usually based on empirical knowledge and subjective judgments by experts at the institutions.

\section{DISCUSSION}

Colors of the original painting and the reproduction postcards may appear similar to our eyes, but colorimetric analysis revealed clear differences between them. Surface reflectances of the postcards of the historical Flemish paintings were much higher than the original painting, which produced the higher lightness and saturation. The distribution of colors in a color space, color gamut, was different between the original painting and the postcard. Depending on the type of the paintings, whether the historic Flemish painting or the modern abstract painting, by Amadeo de Souza-Cardoso, the color gamut of the reproduction postcard is expanded or lessens in comparison to that of the original painting. The effects of the illuminants on the color gamut were observed by the skewness of the color gamut as well as its size.

Color constancy between the original painting and the postcard was quantified by RCCI. The RCCI was relatively high on skin color areas and modest on the nonskin local area with more saturated colors.

\section{A. Consistency of Colors}

In imaging sciences, there are established standards to evaluate the color quality of illuminants, such as color fidelity and CRI, which examine the shifts of colors of specifically defined samples under a test illuminant compared with those under a reference illuminant $[6,30,31]$. Analogous to these methods, it is possible to evaluate a consistency of surface color appearance of the local areas in the original painting and the postcard.

Three types of color differences are being examined. Thus, considering the illuminant D65 as a reference illuminant: (a) color differences at the local areas in the original painting between illuminant D65 and the illuminants with lower CCT; (b) those in the postcards; and (c) color difference at the local areas between the original painting and the postcards under different illuminants.

The ratios of (b) and (c) to (a) indicate whether the shift of colors is consistent between the original painting and the postcard. The ratio unity indicates the color shifts are similar to those within the original painting.

Table 3 shows the ratios of (b)/(a) and (c)/(a) for each painting. The ratios are mostly higher than unity, indicating the color differences or color shifts were larger in the postcards and with the changes in illumination from D65. With the Flemish paintings, the ratio (c)/(a) tended to be higher than the ratio (b)/(a), but it was reversed with the modern paintings.

Taking the average of ratios between the skin and nonskin areas in the Flemish paintings, the two-factor analysis of variance without repetition for the five paintings and four illuminants showed that, for the ratio (b)/(a), each of the effects of the illuminants and paintings was significant $(F(3,12)=29$, $p<0.05 ; F(4,12)=45, p<0.05)$; and for the ratio (c)/ (a), the effect of the illuminants was not significant $(F(3,12)=2.6, p>0.1)$, but the difference between the paintings was significant $(F(4,12)=6.7, p<0.05)$.

\section{B. Relationship between SSV and RCCI}

The colorimetric properties that have been analyzed originate in the spectral properties. It could be argued, therefore, whether the RCCI may be explained by the difference in spectral properties between the original painting and the reproduction postcard. 
Table 3. Color Consistency from Ratios of Color Differences between the Original Paintings and the Postcards ${ }^{a}$

\begin{tabular}{|c|c|c|c|c|c|c|c|c|}
\hline \multirow{3}{*}{$\begin{array}{l}\text { Illuminant } \\
\text { on Postcard }\end{array}$} & \multicolumn{4}{|c|}{ Flemish Painting P1 } & \multicolumn{4}{|c|}{ Flemish Painting P2 } \\
\hline & \multicolumn{2}{|c|}{ Skin } & \multicolumn{2}{|c|}{ Nonskin } & \multicolumn{2}{|c|}{ Skin } & \multicolumn{2}{|c|}{ Nonskin } \\
\hline & $\mathbf{b} / \mathbf{a}$ & c/a & $\mathbf{b} / \mathbf{a}$ & $\mathbf{c} / \mathbf{a}$ & $\mathbf{b} / \mathbf{a}$ & $\mathbf{c} / \mathbf{a}$ & $\mathbf{b} / \mathbf{a}$ & c/a \\
\hline $\mathrm{F} 2$ & 1 & 1. & 1. & 1. & & & 1 & 1. \\
\hline F11 & 1.349 & 1.457 & 1.390 & 1.616 & 1.345 & 1.523 & 1.374 & 1.758 \\
\hline LED & 1.308 & 1.427 & 1.330 & 1.562 & 1.295 & 1.463 & 1.302 & 1.684 \\
\hline D40 & 1.319 & 1.509 & 1.334 & 1.693 & 1.295 & 1.564 & 1.303 & 1.831 \\
\hline
\end{tabular}

\begin{tabular}{|c|c|c|c|c|c|c|}
\hline \multirow{3}{*}{$\begin{array}{l}\text { Illuminant on } \\
\text { Postcard }\end{array}$} & \multicolumn{6}{|c|}{ Modern Painting } \\
\hline & \multicolumn{2}{|c|}{ P172 } & \multicolumn{2}{|c|}{ P200 } & \multicolumn{2}{|c|}{ P38 } \\
\hline & $\mathbf{b} / \mathbf{a}$ & c/a & $\mathbf{b} / \mathbf{a}$ & c/a & $\mathbf{b} / \mathbf{a}$ & c/a \\
\hline$\overline{\mathrm{F}} 2$ & 1.245 & 1.039 & 1.122 & 0.906 & 1.080 & 0.980 \\
\hline F11 & 1.325 & 1.177 & 1.199 & 1.007 & 1.198 & 1.200 \\
\hline LED & 1.290 & 1.220 & 1.164 & 1.565 & 1.157 & 1.111 \\
\hline D40 & 1.327 & 1.206 & 1.183 & 1.737 & 1.176 & 1.086 \\
\hline
\end{tabular}

${ }^{a}$ Ratios of color differences to estimate consistency of colors. (a) Color differences at the local areas in the original painting between illuminant D65 and the illuminants with lower CCT; (b) those in the postcards; and (c) color difference at the local areas between the original painting and the postcards under different illuminants. The ratios of (b) and (c) to (a) indicate whether the shift of colors is consistent between the original painting and the postcard. The ratio unity indicates the color shifts are similar to those within the original painting.

The similarity of spectral properties between the original paintings and the reproduction postcards were quantified by using RMSE and SSV (Subsection 3.A). The RMSE and SSV of the surface reflectances at the selected local areas were regressed to RCCI to evaluate whether the variance of the RCCI may be explained by the variance of RMSE and SSV. These results suggest that the spectral similarity may not be able to explain solely the variance of the RCCI.

\section{Color Preference and Color Memory}

The postcards of the Flemish painting had higher chroma in them than the original when they were observed under the illuminants with the lower CCT (Subsection 3.B).

As discussed, the causes of the chroma shifts and the transformations of color gamut between the original painting and the reproduction postcard may be attributed not only to the type of the paintings but also to the manufacturing process of the reproduction postcard. A conventional protocol of the reproduction process would include the digitization of the artworks, color adjustments and edits, and color printing, at each stage of which color management would be undertaken.

Consider a practical situation where viewers observe artworks at museums; they might memorize hundreds of paintings and remember with the reproduction postcards afterward. In such circumstances, the influence of color memory could contribute to the color adjustments during the manufacturing process.

Shift of saturation of colors in color preference and color memory has been studied extensively. Preference color of the familiar objects and their memory color tend to be remembered as more saturated [18], and color memory of single color patches was shifted to more saturated [19], and categorical focal colors are generally located on saturated region in a color space $[32,33]$. The shift can be extended to the expansion of color gamut or chromaticity caused by the memory of natural scenes (e.g., [15]). Performance of color constancy seemed to be robust with short-term memory of natural scenes $[4,34,35]$. In one of the early studies [15], the expansion of chromatic contrast in natural images was reported by a memory recognition task. Even with involving memory, the color constancy seemed to be robust with a short-term memory of natural scenes $[34,35]$.

However, the expansion by the memory would not be applicable to the modern paintings used in this study, as the color gamut in the reproductions is smaller than the original paintings, where the physical limitation of the color gamut may be taken into account (Subsection 3.D).

Despite these transformations of color gamut and chromatic contrast, the postcard seems to be accepted by the viewers as an alternative to the original painting. This may suggest that the human vision system may have an ability to maintain the constant appreciation of art paintings.

\section{Skin Color Appearance}

Although colors on the skin areas appeared to be "skin," the chromaticity coordinates are in fact less saturated and close to neutral color [see Figs. 6(a) and 7(a)]. Surface color appearance is influenced by their surroundings [36], even if the surrounding surfaces are located remotely [37]. The color appearance of skin areas may be relevant to these phenomena.

The neutral colors would provide robust color constancy $[1,38,39]$, as it may serve as an origin of color shift even with the changes in illuminants. However, the estimation of color constancy by RCCI used in this study is based purely on computation in the local areas. The effect of the surrounding colors was not considered.

As a reference, the color of real Caucasian skin has higher $a^{*}$ and $b^{*}$ chromaticities than those in the Flemish paintings and the corresponding postcards. The mean $\left(a^{*}, b^{*}\right)$ chromaticity coordinates in the CIELAB are approximately $\left(a^{*}, b^{*}\right)=$ $(12,15)[26]$.

\section{CONCLUSION}

Spectral and colorimetric analyses were undertaken to assess color constancy on the reproduction postcards in relevance to the original paintings of the 15th-century Flemish paintings and the 20th-century modern abstract paintings by Amadeo de Souza-Cardoso. Reproduction color constancy across the daylight D65 and the common illuminants with lower CCT of approximately $4000 \mathrm{~K}$ was assessed by the RCCI. The RCCI was reasonably high, which was comparable to the human color constancy in psychophysics but varied depending on the colors in the local areas. The types of the paintings and illuminants influenced the statistics of color properties, such as the transformations of color gamut. The results suggest that viewers may be able to accept the reduction of color accuracy when illuminations were altered and to achieve good color 
appreciation of the original art paintings with the reproduction postcards.

Funding. European Cooperation in Science and Technology (COST), Action TD1201, Colour and Space in Cultural Heritage (COSCH): Short Term Scientific Missions (COST-STSM-TD1201-14213).

Acknowledgment. The authors thank Museu Nogueira Da Silva, Portugal, and Centro de Arte Moderna da Fundação Gulbenkian, Lisboa, Portugal, for their cooperation. The authors thank Osamu Masuda and Hélder Tiago Correia, and Cristina Montagner for helping in the spectral imaging of the paintings and thank David H. Foster for the use of the hyperspectral imaging system.

\section{REFERENCES}

1. D. H. Foster, "Color constancy," Vis. Res. 51, 674-700 (2011).

2. S. M. C. Nascimento, J. M. M. Linhares, C. Montagner, C. A. R. João, K. Amano, C. Alfaro, and A. Bailão, "The colors of paintings and viewers' preferences," Vis. Res. 130, 76-84 (2017).

3. D. H. Foster, K. Amano, and S. M. C. Nascimento, "Color constancy in natural scenes explained by global image statistics," Vis. Neurosci. 23, 341-349 (2006).

4. K. Amano and D. H. Foster, "Effect of spatial uncertainty and familiarity on memory for surface colour in natural scenes and Mondrian patterns," in Conference on Colour in Graphics, Imaging, and Vision 2008 (2008), pp. 223-226.

5. C. Montagner, "The brushstroke and materials of Amadeo de SouzaCardoso combined in an authentication tool," Ph.D. dissertation (Faculdade de Ciências e Tecnologia, Universidade NOVA de Lisboa, 2015).

6. Illuminating Engineering Society, "IES method for evaluating light source color rendition," TM-30-15 (2015).

7. M. Scuello, I. Abramov, J. Gordon, and S. Weintraub, "Museum lighting: optimizing the illuminant," Color Res. Appl. 29, 121-127 (2004).

8. D. Garside, K. Curran, C. Korenberg, L. MacDonald, K. Teunissen, and S. Robson, "How is museum lighting selected? an insight into current practice in UK museums," J. Inst. Conserv. 40, 3-14 (2017).

9. S. M. C. Nascimento and O. Masuda, "Best lighting for visual appreciation of artistic paintings-experiments with real paintings and real illumination," J. Opt. Soc. Am. A 31, A214-A219 (2014).

10. F. Viénot, G. Coron, and B. Lavédrine, "LEDs as a tool to enhance faded colours of museums artefacts," J. Cult. Herit. 12, 431-440 (2011).

11. L. E. Arend, A. Reeves, J. Schirillo, and R. Goldstein, "Simultaneous color constancy: papers with diverse Munsell values," J. Opt. Soc. Am. A 8, 661-672 (1991).

12. A. J. Reeves, K. Amano, and D. H. Foster, "Color constancy: phenomenal or projective?" Percept. Psychophys. 70, 219-228 (2008).

13. K. Amano, A. Sohaib, K. Xiao, J. Yates, C. Whitford, and S. Wuerger, "Color quality assessments of 3D facial prostheses in varying illuminations," J. Vis. 17(10), 138 (2017).

14. A. Sohaib, K. Amano, K. Xiao, J. M. Yates, C. Whitford, and S. Wuerger, "Colour quality of facial prostheses in additive manufacturing," Int. J. Adv. Manuf. Technol. (to be published), doi:10.1007/ s00170-017-1480-x.

15. K. Amano, K. Uchikawa, and I. Kuriki, "Characteristics of color memory for natural scenes," J. Opt. Soc. Am. A 19, 1501-1514 (2002).
16. CIE, "Guidelines for the evaluation of gamut mapping algorithms," $\mathrm{CIE}$ 156:2004 (Commission Internationale de l'Éclairage, 2004).

17. J. Morovič, Color Gamut Mapping (Wiley, 2008).

18. C. J. Bartleson, "Memory colors of familiar objects," J. Opt. Soc. Am. 50, 73-77 (1960).

19. S. M. Newhall, R. W. Burnham, and J. R. Clark, "Comparison of successive with simultaneous color matching," J. Opt. Soc. Am. 47, 43-56 (1957).

20. F. de Helena, A. Cardoso, M. J. Melo, M. Vilarigues, S. Babo, and C. Alfaro, Catálogo Raisonné Amadeo de Souza-Cardoso, Pintura (Fundação Calouste Gulbenkian, 2008).

21. C. Montagner, J. M. M. Linhares, M. Vilarigues, M. J. Melo, and S. M. C. Nascimento, "Supporting history of art with colorimetry: the paintings of Amadeo de Souza-Cardoso," Color Res. Appl. (to be published).

22. D. H. Foster, K. Amano, S. M. C. Nascimento, and M. J. Foster, "Frequency of metamerism in natural scenes," J. Opt. Soc. Am. A 23, 2359-2372 (2006).

23. D. B. Judd, D. L. MacAdam, G. Wyszecki, H. W. Budde, H. R. Condit, S. T. Henderson, and J. L. Simonds, "Spectral distribution of typical daylight as a function of correlated color temperature," J. Opt. Soc. Am. 54, 1031-1040 (1964).

24. R. Shrestha, R. Pillay, S. George, and J. Y. Hardeberg, "Quality evaluation in spectral imaging-quality factors and metrics," J. Int. Colour Assoc. 12, 22-35 (2014).

25. Rochester Institute of Technology, "Program of color science: useful color data," https://www.rit.edu/science/pocs/useful-data.

26. K. Xiao, J. M. Yates, F. Zardawi, S. Sueeprasan, N. Liao, L. Gill, C. Li, and S. Wuerger, "Characterising the variations in ethnic skin colours: a new calibrated data base for human skin," Skin Res. Technol. 23, 21-29 (2017).

27. D. Nickerson, "Light sources and color rendering," J. Opt. Soc. Am. 50, 57-69 (1960).

28. S. M. C. Nascimento, F. P. Ferreira, and D. H. Foster, "Statistics of spatial cone-excitation ratios in natural scenes," J. Opt. Soc. Am. A 19, 1484-1490 (2002).

29. International Organization for Standardization, "Image technology colour management - Architecture, profile format and data structure Part 1: Based on ICC.1:2010," ISO 15076-1:2010 (2010).

30. M. S. Rea and J. P. Freyssinier-Nova, "Color rendering: a tale of two metrics," Color Res. Appl. 33, 192-202 (2008).

31. CIE, "Method of measuring and specifying colour rendering properties of light sources," CIE 013.3-1995 (Commission Internationale de l'Éclairage, 1995).

32. R. M. Boynton and C. X. Olson, "Locating basic colors in the OSA space," Color Res. Appl. 12, 94-105 (1987).

33. K. Uchikawa and H. Shinoda, "Influence of basic color categories on color memory discrimination," Color Res. Appl. 21, 430-439 (1996).

34. Y. Ling and A. Hurlbert, "Role of color memory in successive color constancy," J. Opt. Soc. Am. A 25, 1215-1226 (2008).

35. E. W. Jin and S. K. Shevell, "Color memory and color constancy," J. Opt. Soc. Am. A 13, 1981-1991 (1996).

36. R. O. Brown and D. I. A. MacLeod, "Color appearance depends on the variance of surround colors," Curr. Biol. 7, 844-849 (1997).

37. P. Monnier and S. K. Shevell, "Large shifts in color appearance from patterned chromatic backgrounds," Nat. Neurosci. 6, 801-802 (2003).

38. J. M. Speigle and D. H. Brainard, "Predicting color from gray: the relationship between achromatic adjustment and asymmetric matching," J. Opt. Soc. Am. A 16, 2370-2376 (1999).

39. J. M. Speigle and D. H. Brainard, "Luminosity thresholds: effects of test chromaticity and ambient illumination," J. Opt. Soc. Am. A 13 436-451 (1996). 